\title{
Editorial: Cutting-Edge Metastructures: Micro-Architected and Active Metamaterials
}

\author{
D. Misseroni ${ }^{1 *}$, A. Bacigalupo ${ }^{2}$, M. L. De Bellis ${ }^{3}$, A. B. Movchan ${ }^{4}$ and G. H. Paulino ${ }^{5}$ \\ ${ }^{1}$ DICAM, University of Trento, Trento, Italy, ${ }^{2} D I C C A$, University of Genova, Genova, Italy, ${ }^{3}$ INGEO, University of Chieti-Pescara, \\ Chieti, Italy, ${ }^{4}$ Department of Mathematical Sciences, University of Liverpool, Liverpool, United Kingdom, ${ }^{5}$ School of Civil and \\ Environmental Engineering, Georgia Institute of Technology, Atlanta, GA, United States
}

Keywords: lattice materials, mechanical metamaterials, seismic metamaterials, active metamaterials, cloaking, relaxed micromorphic modeling, gyroscopic spinners, signal attenuation

Editorial on the Research Topic

Cutting-Edge Metastructures: Micro-Architected and Active Metamaterials

The present Research Topic collects articles dealing with the optimal design, the realization and the testing of meta-structures capable of achieving dynamic high-performance. The common thread linking these articles is using metamaterials concepts to conceive intriguing internal architecture for wave propagation control. Due to their interdisciplinary nature and their broad range of applications, metamaterials have increasingly inspired researchers of different communities. In the last years, mathematicians, physicists, engineers, and material scientists have mobilized massive effort to move the limits beyond state-of-the-art to realize extreme materials. Charming behaviors such as filtering, wave-guiding, negative refraction, cloaking, seismic protection, noise reduction and band-gap control have been demonstrated to be possible theoretically, via numerical simulations and by real experiments. Different techniques have been developed for achieving such exotic effects. A nonexhaustive list of these includes i) mass, inertia and stiffness modifications, ii) the use of local inertial or Helmholtz resonators or gyroscopes elements embedded into lattice or continuum materials, iii) the use of a multi-field electro-mechanical coupling via piezoelectric active phases. Within this framework, the current research topics of the articles provide a new perspective for using the metamaterial idea to achieve phenomena such as cloaking, seismic protection, dynamic wave propagation attenuation, and wave localization.

The present collection includes six research articles and one review article. The review article by Yakovleva et al., provides an overview of the main theories used to describe the response of microstructured elastic solids subjected to dynamic loads. Some examples of wave propagation in structured elastic media are presented, such as applying metamaterial concepts to describe the dynamics of real-life bridges. Particular emphasis is given to the reliability of theoretical models, derived considering an infinite structure, to describe dynamic of finite span elements. The links between flexural metamaterials, metasurfaces, and predictive seismic assessments are discussed through the case study of a region affected by an earthquake.

The performance of large-scale metamaterials in seismic protection and noise reduction is discussed by Varma et al.. Here the focus is on investigating the effect of some parameters, such as microstructure geometry, orientation, constraints and constituent materials, in opening band gaps at low frequency regimes. The numerical analysis show that not only steel but also concrete inclusions can lead to large wave attenuation. A design of a protective barrier for civil engineering constructions is proposed and discussed.

Potential applications in seismic shielding and wave scattering reduction have the outcomes reported in the article by Rossi et al.. This work is devoted to investigating some relevant issues encountered in the 
realization of flexural cloaks via a regularized near-cloak transformation, as the role of bending stiffness and twisting rigidity on the behavior of the meta-structural plate. The performance of the elastic cloak for transient flexural waves is estimated by a parametric analysis carried out with an in-house Finite Element code. The extension of the general theory of thinplate cloaks to the case of its interaction with a Winkler foundation is analyzed theoretically and then verifi/ed via numerical simulations.

The article by Nieves et al. investigates the propagation of Rayleigh waves into a semi-infinite gyroscopes triangular lattice. The Rayleigh waves are induced by a point load acting at the boundary of the lattice. The analytical expression of the displacement field is derived and compared with the results obtained from the numerical simulations. A detailed parametric study is performed to capture the influence of different physical quantities (direction and radian frequency of the force, gyricity of the spinners) on the behavior of the gyroscopic system. This work reveals that fascinating effects, such as energy splitting and directionality in propagating waves can be achieved.

A different approach, namely a multifield electro-mechanical method, is exploited by Dorin et al. for the control of the wave propagation. In the article, the authors propose a piezoelectric metamaterial capable of tailoring simultaneously frequency, path, and mode shape of topological waves using resonant circuitry. In addition to its on-demand tunability, it is shown, by systematic numerical simulations, that the proposed metamaterial operates over a broad frequency bandwidth. The authors suggest that their results can find applications in the design of vibration isolators, wave filters, and energy harvesters.

Rizzi et al. deals with the open topic of anomalous refraction in a meta-structure where a metamaterial is embedded into an ordinary homogeneous material. The refractive properties of a metamaterial interacting with other materials are investigated via the relaxed micromorphic model. The analytical and numerical results reported in the article show the great versatility of the proposed configuration, which could provide several different non-trivial effects. For instance, a change in the stiffness of specific regions of a homogeneous material in contact with a metamaterial slab can lead to radically different behavior, e.g. acoustic screen or acoustic absorber.

The metamaterial idea is also exploited by Briccola et al. to enhance wave attenuation in concrete with quasi-randomly arranged inclusions. The inclusions are spherical and consist of a heavy central core coated with a soft material. A specific experimental campaign is carried out to quantify the attenuation properties of metaconcrete for dynamic waves in the sonic range. This study shows that a combination of different types of engineered aggregates within the matrix can enhance the attenuation capabilities of the metaconcrete.

The variety of the contributions collected in this research topic is proof of the cross-disciplinary nature of mechanical metamaterials. The insights emerging from these articles can be undoubtedly the starting point and the inspiration for further developments. The almost endless applications of metamaterial concepts, highlighted by the collected papers, suggest that the metamaterials research field is an open topical theme with a vast potential still to be explored.

\section{AUTHOR CONTRIBUTIONS}

All authors listed have made a substantial, direct, and intellectual contribution to the work and approved it for publication.

\section{FUNDING}

DM is supported by the European Commission under the H2020 FET Open ("Boheme") grant No. 863179. DM and AB are supported by University of Trento under the project UNMASKED 2020. AB is supported by the Compagnia San Paolo under the project MINIERA no. I34I20000380007. MDB is supported by the project Search for Excellence Ud'A 2019.

\section{ACKNOWLEDGMENTS}

We would like to thank all the reviewers and the authors whose contributions have been fundamental to make a successful research topic collection.

Conflict of Interest: The authors declare that the research was conducted in the absence of any commercial or financial relationships that could be construed as a potential conflict of interest.

Copyright (c) 2021 Misseroni, Bacigalupo, De Bellis, Movchan and Paulino. This is an open-access article distributed under the terms of the Creative Commons Attribution License (CC BY). The use, distribution or reproduction in other forums is permitted, provided the original author(s) and the copyright owner(s) are credited and that the original publication in this journal is cited, in accordance with accepted academic practice. No use, distribution or reproduction is permitted which does not comply with these terms. 